\title{
Genome-wide detection of genetic markers associated with growth and fatness in four pig populations using four approaches
}

\author{
Yuanmei Guo ${ }^{\dagger}$, Yixuan Huang ${ }^{\dagger}$, Lijuan Hou, Junwu Ma, Congying Chen, Huashui Ai, Lusheng Huang \\ and Jun Ren ${ }^{*}$ (D)
}

\begin{abstract}
Background: Genome-wide association studies (GWAS) have been extensively used to identify genomic regions associated with a variety of phenotypic traits in pigs. Until now, most GWAS have explored single-trait association models. Here, we conducted both single- and multi-trait GWAS and a meta-analysis for nine fatness and growth traits on 2004 pigs from four diverse populations, including a White Duroc $\times$ Erhualian $F_{2}$ intercross population and Chinese Sutai, Laiwu and Erhualian populations.

Results: We identified 44 chromosomal regions that were associated with the nine traits, including four genomewide significant single nucleotide polymorphisms (SNPs) on SSC2 (SSC for Sus scrofa chromosome), 4, 7 and X. Compared to the single-population GWAS, the meta-analysis was less powerful for the identification of SNPs with population-specific effects but more powerful for the detection of SNPs with population-shared effects. Multiple-trait analysis reduced the power to detect trait-specific SNPs but significantly enhanced the power to identify common SNPS across traits. The SNP on SSC7 had pleiotropic effects on the nine traits in the $F_{2}$ and Erhualian populations. Another pleiotropic SNP was observed on SSCX for these traits in the $F_{2}$ and Sutai populations. Both populationspecific and shared SNPs were identified in this study, thus reflecting the complex genetic architecture of pig growth and fatness traits.
\end{abstract}

Conclusions: We demonstrate that the multi-trait method and the meta-analysis on multiple populations can be used to increase the power of GWAS. The two significant SNPs on SSC7 and X had pleiotropic effects in the $F_{2}$, Erhualian and Sutai populations.

\section{Background}

Growth and fatness traits are economically important and have been intensively selected in the global pig industry. Dissection of the genetic architecture of growth and fat deposition in pigs not only benefits the pig industry but also sheds insight into our understanding of human obesity, because pigs are more physiologically similar to humans than rodents and other model animals [1].

To understand the molecular basis of divergent phenotypes in pigs, researchers have established multiple

\footnotetext{
*Correspondence: renjunjxau@hotmail.com

†Yuanmei Guo and Yixuan Huang contributed equally to this work State Key Laboratory of Pig Genetic Improvement and Production

Technology, Jiangxi Agricultural University, Nanchang 330045, China
}

$\mathrm{F}_{2}$ intercross populations using European and Chinese breeds as founders, and have mapped quantitative trait loci (QTL) for a list of phenotypic traits, including growth and fatness traits, using hundreds of microsatellite markers across the whole genome [2-6]. Until now, 1880 and 1070 QTL for growth and fatness traits have been deposited in the pig QTL database (http://www.animalgenome. org/cgi-bin/QTLdb/SS/index, Release 26, Apr 27, 2015), respectively. These findings have significantly advanced our understanding of the genetic architecture of porcine growth and fatness traits. Nevertheless, the resolution of traditional QTL mapping is relatively poor due to markers being sparse and insufficient recombination events in 
the $F_{2}$ crosses. Thus, identification of causative mutations that underlie the identified QTL remains a big challenge.

With the availability of the Illumina Porcine SNP60 Beadchip, it has become feasible to exploit the association between high-density single nucleotide polymorphisms (SNPs) and phenotypic traits through genome-wide association studies (GWAS) [7]. Compared to the traditional QTL mapping approach, the GWAS approach allows the identification of SNPs that are significantly associated with traits. Nevertheless, large sample sizes are still required to identify SNPs that are weakly associated with target traits. A meta-analysis of GWAS can not only increase statistical power but also reduce the number of false positives by combining information from multiple independent studies [8]. Moreover, for a QTL with pleiotropic effects on multiple traits, a multi-trait analysis can improve the detection power of GWAS [9-11].

The aim of our study was to identify SNPs associated with nine traits related to growth and fatness across four pig populations, including a White Duroc $\times$ Erhualian $\mathrm{F}_{2}$ intercross (referred hereafter as $\mathrm{F}_{2}$ ), Sutai, Laiwu and Erhualian pigs by four GWAS methods: single-trait analysis on a single population (SS-GWAS), single-trait analysis on multiple populations (SM-GWAS), multi-trait analysis on a single population (MS-GWAS), and multitrait analysis on multiple populations (MM-GWAS).

\section{Methods}

\section{Ethics statement}

All procedures used for this study and involving animals are in compliance with guidelines for the care and utility of experimental animals established by the Ministry of Agriculture of China. The ethics committee of Jiangxi Agricultural University specifically approved this study.

\section{Animals and phenotypic measurements}

A total of 2004 pigs were used in this study, including 925, 434, 331 and 314 individuals from the $F_{2}$, Sutai, Erhualian and Laiwu populations, respectively. The $F_{2}$ and Sutai populations were previously described in $[12,13]$. Briefly, the $F_{2}$ animals originated from a cross between two White Duroc boars and 17 Erhualian sows [12]. In this population, nine $F_{1}$ boars and $59 F_{1}$ sows were intercrossed by avoiding full-sib mating to produce $1912 \mathrm{~F}_{2}$ pigs. A total of $925 \mathrm{~F}_{2}$ pigs were randomly selected from all $F_{1}$ boar and sow families. These animals were slaughtered at the age of $240 \pm 3$ days and carcass and meat quality traits were measured; the remaining $F_{2}$ pigs were used to produce $\mathrm{F}_{3}$ individuals or measure the reproductive traits. The Sutai pig is a Chinese synthetic breed that was derived from a cross between Western Duroc boars and Chinese Taihu (mainly Erhualian) sows. This breed has experienced directional selection for prolificacy and growth for more than 18 generations. The 434 Sutai pigs used in the current study were offspring of four sires and 55 dams [13]. Erhualian and Laiwu are Chinese indigenous breeds. The former is known for its prolificacy, with a litter size that can exceed 15 , and the latter is characterized by its exceptionally high intramuscular fat content (more than 9\%) [14]. We obtained 334 Erhualian (168 sires and 166 dams) and 314 Laiwu (218 sires and 98 dams) pigs at the age of $\sim 90$ days from two national conservation farms of the two breeds in Jiangsu and Shandong provinces, respectively. The $\mathrm{F}_{2}$, Sutai, Erhualian and Laiwu pigs were all raised in an experimental farm in Nanchang, Jiangxi province from 2001 to 2014. All 2004 pigs had ad libitum access to fresh water and consistent feed containing $16 \%$ crude protein, $3100 \mathrm{~kJ}$ of digestive energy, and $0.78 \%$ lysine during the fattening period. Each pig in the four populations was weighed at birth and at 210 and 240 days of age, and the average daily gains from 0 to 210 days of age $\left(\mathrm{ADG}_{0-210}\right)$ and from 210 to 240 days of age ( $\left.\mathrm{ADG}_{210-240}\right)$ were calculated. The $\mathrm{F}_{2}$ and Sutai pigs were slaughtered in the same commercial abattoir at $240 \pm 3$ days of age and the Erhualian and Laiwu pigs at $300 \pm 3$ days of age. After slaughter, all pigs were measured for fatness traits, including backfat thickness at the shoulder (SBF), the first rib (FBF), the last rib (LBF), and at the hip (HBF), and weight of leaf fat (LFW), veil fat (VFW) and abdominal fat (AFW).

\section{Genotyping and quality control}

Genomic DNA was extracted from ear or tail tissues using a standard phenol/chloroform protocol, and was then quantified and adjusted to a final concentration of $50 \mathrm{ng} / \mu \mathrm{l}$. All 2004 pigs were genotyped with the porcine $60 \mathrm{~K}$ SNP Beadchip on an iScan System (Illumina, USA) following the manufacturer's protocol. SNPs, including sex-linked SNPs, that had a call rate less than $95 \%$, a minor allele frequency lower than $5 \%$, or that strongly deviated from Hardy-Weinberg equilibrium $(P<0.000001)$ were discarded. Animals with a call rate less than 95\% were also removed from further analyses. These quality controls were performed for each population separately to include as many qualified SNPs as possible for the GWAS in each population. A common set of 15,429 qualified SNPs across the four populations was used in the meta-analysis of GWAS.

\section{Statistical methods}

Descriptive statistics of phenotypic traits were calculated by the MEANS procedure of SAS9.0 (SAS Institute Inc., USA) and phenotypic differences between sexes were tested by the TTEST procedure. The MIXED procedure was used to determine the fixed effects and the covariates included in the GWAS model. Sex and fattening batch 
were included as fixed effects in all GWAS models, and birth weight, body weight at 210 days, and carcass weight were treated as covariates for $\mathrm{ADG}_{0-210}, \mathrm{ADG}_{210-240}$ and for fatness traits, respectively, in the GWAS models. A polygenic effect for each animal with covariances based on genomic kinship, which was calculated based on the identity-by-state of the SNPs on autosomes $[15,16]$, was included as a random effect to account for the effect of population substructure. The $P$ values of Bonferroni corrected thresholds for suggestive, 5 and $1 \%$ genome-wide significant levels were $1,0.05$ and 0.01 , respectively, divided by the number of SNPs used in the GWAS. The suggestive level was first proposed by Lander and Kruglyak [17] and represents the threshold where, under the null hypothesis, one false positive is expected per genome scan.

\section{GWAS for a single trait in a single population (SS-GWAS)}

The two-stage approach implemented in the $\mathrm{R}$ package GenABEL was used to conduct SS-GWAS under an additive model. First, the following mixed model was used to calculate the phenotypic residual vector $\mathbf{e}^{*}$ :

$$
\mathbf{y}=\mathbf{X b}+\mathbf{Z u}+\mathbf{e}^{*},
$$

where $\mathbf{y}$ is a vector of phenotypes; $\mathbf{b}$ is the estimator vector of fixed effects, including population mean $\mu$; $\mathbf{u}$ is a vector of random polygenic additive effects that follows a normal distribution $N\left(\mathbf{0}, \mathbf{G} \sigma_{\mathrm{u}}^{2}\right)$, with $\mathbf{G}$ being the genomic kinship matrix calculated from all autosomal SNPs based on identity-by-state [16], $\sigma_{\mathrm{u}}^{2}$ is the polygenic additive variance, and $\mathbf{X}$ and $\mathbf{Z}$ are the incidence matrices for $\mathbf{b}$ and $\mathbf{u}$, respectively.

Then, a family-based score test was used to detect associations between SNPs and traits using the following simple regression model [18], one SNP at a time:

$$
\mathbf{e}^{*}=\mathbf{S a}+\mathbf{e},
$$

where a is an estimator of the SNP allele substitution effect; $\mathbf{S}$ is the incidence vector for a (coded 0, 1, 2 based on allele dosage); and $\mathbf{e}$ is a vector of residual errors that follows a normal distribution $N\left(\mathbf{0}, \mathbf{I} \sigma_{\mathrm{e}}^{2}\right)$, with $\mathbf{I}$ being an identity matrix and $\sigma_{\mathrm{e}}^{2}$ the variance of the residual error. The $P$ value of the association test was adjusted by the genomic control method to correct for residual inflation $[19,20]$.

\section{GWAS for multiple traits in a single population (MS-GWAS)}

MS-GWAS were conducted to detect pleiotropic SNPs using the method proposed by Bolormaa et al. [11]. In brief, a Chi square statistic, which approximately follows a Chi square distribution with the number of traits tested as the number of degrees of freedom, was calculated for each SNP using the following formula:

$$
\chi_{\text {multi-trait }}^{2}=\mathbf{t}_{i}^{\prime} \mathbf{V}^{-1} \mathbf{t}_{i},
$$

where $\mathbf{t}_{i}$ is a $9 \times 1$ vector of the signed $t$-values for the $i$ th SNP from the SS-GWAS for the nine traits, $\mathbf{t}_{i}^{\prime}$ is the transpose of $\mathbf{t}_{i}$, and $\mathbf{V}^{-1}$ is the inverse of the $9 \times 9$ correlation matrix between traits. The correlation between two traits was calculated by correlating the estimated effects (signed $t$-values) of the 15,429 qualified SNPs for the two traits.

\section{Meta-analysis of GWAS for a single trait (SM-GWAS) and multiple traits (MM-GWAS)}

We applied the inverse variance weighting method of [8], in which each population is weighted according to the inverse of its squared standard error, to perform SM-GWAS and MM-GWAS across the four populations based on the results of SS-GWAS and MS-GWAS, respectively. The weight $\left(w_{i}\right)$ for the $i$ th population was equal to the inverse of the square of the standard error $\left(s_{i}\right)$ of the allele substitution effect in the $i$ th population. Then, the pooled estimates of the allele effect $(\beta)$ of a given SNP and its standard error $(s)$ were calculated as follows:

$$
\beta=\frac{\sum_{i=1}^{n} w_{i} \beta_{i}}{\sum_{i=1}^{n} w_{i}} \text { and } s^{2}=\frac{1}{\sum_{i=1}^{n} w_{i}},
$$

where $n$ is the population number; $\beta_{i}$ is the allele effect in the $i$ th population.

A statistic ( $Z$ score) of $Z$-test was calculated as follows:

$$
Z=\frac{\beta}{s}=\frac{\sum_{i=1}^{n} w_{i} \beta_{i}}{\sqrt{\sum_{i=1}^{n} w_{i}}}
$$

An allele of an associated SNP may have a positive effect in some populations and a negative effect in the others. Such an inconsistent effect could significantly reduce the detection power of the meta-analysis of GWAS. To circumvent this, we used information on linkage disequilibrium and ignored information on phase, i.e., the absolute value of $\beta_{i}$ was used to calculate the pooled $\beta$ and $Z$ values.

To determine if a SNP that was significantly associated with multiple traits was due to closely linked genes or pleiotropy, we conducted a conditional SS-GWAS by fixing the effect of the top SNPs identified by MS-GWAS in the statistic model.

\section{Linkage and linkage disequilibrium analyses}

To determine the approximate genomic positions of the unmapped significant SNPs, a two-point linkage analysis was used to detect linkage between the mapped and unmapped SNPs in the $F_{2}$ population [21]. The lower the recombination rate $(\theta)$ is, the tighter is the link 
between the two SNPs. Haplotypes of the regions that were significantly associated with the target traits were inferred by Simwalk2.9 [22], and linkage disequilibrium blocks were defined using default parameters of Haploview4.2 [23]. The VennDiagram in R package was used to draw a Venn diagram that showed the loci that were

Table 1 Genome-wide significant loci for nine fatness and growth traits identified by four GWAS approaches in this study

\begin{tabular}{|c|c|c|c|c|c|c|c|c|c|c|c|}
\hline $\mathrm{QTL}^{\mathrm{a}}$ & $C h r^{b}$ & Trait $^{c}$ & Population & Method & Top SNP & Pos $\mathrm{Mb}^{\mathrm{d}}$ & Effect $\pm S E^{e}$ & $P$ value $^{f}$ & $N_{\text {SNP }}^{g}$ & Boundary SN & \\
\hline 1 & 2 & HBF & Meta & SM & ss131211507 & 3.61 & $0.127 \pm 0.026$ & $1.28 \mathrm{E}-06^{*}$ & 1 & - & - \\
\hline 2 & 4 & Multi-trait & Meta & MM & ss131269439 & 81.71 & - & $7.88 \mathrm{E}-10^{* *}$ & 8 & ss131269439 & ss478935222 \\
\hline 2 & 4 & FBF & Meta & SM & ss131269439 & 81.71 & $0.168 \pm 0.032$ & $1.20 \mathrm{E}-07^{* *}$ & 1 & - & - \\
\hline 2 & 4 & $\mathrm{HBF}$ & Meta & SM & ss131269678 & 82.25 & $0.156 \pm 0.032$ & $1.08 \mathrm{E}-06^{*}$ & 1 & - & - \\
\hline 2 & 4 & SBF & Meta & SM & ss478935222 & 85.09 & $0.166 \pm 0.035$ & $1.53 \mathrm{E}-06^{* *}$ & 1 & - & - \\
\hline 3 & 6 & $\mathrm{ADG}_{210-240}$ & Meta & SM & ss131029816 & 71.60 & $0.040 \pm 0.008$ & $1.38 \mathrm{E}-06^{*}$ & 1 & - & - \\
\hline 4 & 7 & $\mathrm{ADG}_{210}$ & $\mathrm{~F}_{2}$ & SS & ss131342496 & 32.96 & $-0.026 \pm 0.005$ & $5.95 \mathrm{E}-07^{*}$ & 1 & - & - \\
\hline 4 & 7 & Multi-trait & Meta & MM & ss131343534 & 34.56 & - & $2.65 E-18^{* *}$ & 27 & ss120018804 & ss131348342 \\
\hline 4 & 7 & SBF & Meta & SM & ss131343534 & 34.56 & $0.255 \pm 0.042$ & $1.11 \mathrm{E}-09^{* *}$ & 6 & ss131341589 & ss107890951 \\
\hline 4 & 7 & FBF & Meta & SM & ss131343534 & 34.56 & $0.315 \pm 0.040$ & $4.88 \mathrm{E}-15^{* *}$ & 17 & ss131341589 & ss131348342 \\
\hline 4 & 7 & $\mathrm{HBF}$ & Meta & SM & ss131343534 & 34.56 & $0.379 \pm 0.045$ & $3.16 \mathrm{E}-17^{* *}$ & 25 & Ss120018804 & ss131348342 \\
\hline 4 & 7 & Multi-trait & $\mathrm{F}_{2}$ & MS & ss107837325 & 34.80 & - & $3.97 \mathrm{E}-33^{* *}$ & 107 & ss23131766 & ss478941636 \\
\hline 4 & 7 & AFW & $\mathrm{F}_{2}$ & SS & ss107837325 & 34.80 & $0.163 \pm 0.022$ & $8.99 E-11^{* *}$ & 34 & ss131066868 & ss131345041 \\
\hline 4 & 7 & FBF & $\mathrm{F}_{2}$ & SS & ss107837325 & 34.80 & $0.691 \pm 0.060$ & $3.10 E-17^{* *}$ & 69 & ss131341676 & ss131348342 \\
\hline 4 & 7 & $\mathrm{HBF}$ & $\mathrm{F}_{2}$ & SS & ss107837325 & 34.80 & $0.773 \pm 0.072$ & $1.97 \mathrm{E}-14^{* *}$ & 64 & ss131341676 & ss131347489 \\
\hline 4 & 7 & LBF & $F_{2}$ & SS & ss 107837325 & 34.80 & $0.571 \pm 0.057$ & $1.77 \mathrm{E}-14^{* *}$ & 60 & ss131341676 & ss131348342 \\
\hline 4 & 7 & LFW & $F_{2}$ & SS & ss107837325 & 34.80 & $0.650 \pm 0.058$ & $1.75 E-18^{* *}$ & 63 & ss131341766 & ss131347489 \\
\hline 4 & 7 & SBF & $\mathrm{F}_{2}$ & SS & ss107837325 & 34.80 & $0.487 \pm 0.057$ & $1.94 \mathrm{E}-12^{* *}$ & 45 & ss131341766 & ss131346335 \\
\hline 4 & 7 & Multi-trait & Erhualian & MS & ss131343870 & 34.84 & - & $1.29 \mathrm{E}-15^{* *}$ & 6 & ss131342502 & ss131347459 \\
\hline 4 & 7 & FBF & Erhualian & SS & ss131343870 & 34.84 & $0.585 \pm 0.073$ & $2.13 E-13^{* *}$ & 8 & ss131336720 & ss131347459 \\
\hline 4 & 7 & $\mathrm{HBF}$ & Erhualian & SS & ss131343870 & 34.84 & $0.502 \pm 0.075$ & $1.49 \mathrm{E}-10^{* *}$ & 5 & ss131343870 & ss131347459 \\
\hline 4 & 7 & LBF & Erhualian & SS & ss131343870 & 34.84 & $0.523 \pm 0.064$ & $6.96 \mathrm{E}-14^{* *}$ & 5 & ss131343870 & ss131347459 \\
\hline 4 & 7 & LFW & Erhualian & SS & ss131343870 & 34.84 & $0.453 \pm 0.067$ & $2.39 \mathrm{E}-10^{* *}$ & 5 & ss131343870 & ss131347459 \\
\hline 4 & 7 & SBF & Erhualian & SS & ss131343870 & 34.84 & $0.453 \pm 0.079$ & $4.08 \mathrm{E}-08^{* *}$ & 2 & ss131343870 & ss478941599 \\
\hline 4 & 7 & LFW & Meta & SM & ss131344553 & 36.20 & $0.140 \pm 0.028$ & $7.18 \mathrm{E}-07^{*}$ & 2 & ss131344553 & ss131347175 \\
\hline 4 & 7 & $\mathrm{LBF}$ & Meta & SM & ss131347175 & 40.85 & $0.155 \pm 0.024$ & $1.27 \mathrm{E}-10^{* *}$ & 13 & ss131337529 & ss131348342 \\
\hline 5 & $x$ & SBF & Meta & SM & ss131570179 & 46.75 & $0.124 \pm 0.020$ & $5.42 \mathrm{E}-10^{* *}$ & 5 & ss131036304 & ss131562987 \\
\hline 5 & $x$ & VFW & $\mathrm{F}_{2}$ & SS & ss107834496 & 51.70 & $0.091 \pm 0.013$ & $5.39 \mathrm{E}-10^{* *}$ & 21 & Ss478944418 & ss478935791 \\
\hline 5 & $x$ & Multi-trait & $\mathrm{F}_{2}$ & MS & ss23131102 & 63.65 & - & $5.78 \mathrm{E}-35^{* *}$ & 79 & ss131067158 & ss131563360 \\
\hline 5 & $x$ & FBF & $\mathrm{F}_{2}$ & SS & ss23131102 & 63.65 & $0.164 \pm 0.023$ & $2.94 \mathrm{E}-07^{*}$ & 2 & ss478943984 & ss23131102 \\
\hline 5 & $x$ & $\mathrm{HBF}$ & $\mathrm{F}_{2}$ & SS & ss23131102 & 63.65 & $0.278 \pm 0.029$ & $6.81 E-12^{* *}$ & 24 & ss478936157 & ss131562911 \\
\hline 5 & $x$ & Multi-trait & Sutai & MS & ss478934917 & 78.58 & - & $3.19 E-10^{* *}$ & 38 & ss478944000 & ss131570171 \\
\hline 5 & $x$ & Multi-trait & Meta & MM & ss131070541 & 106.48 & - & $1.61 \mathrm{E}-15^{* *}$ & 14 & ss478936157 & ss131562987 \\
\hline 5 & $x$ & FBF & Meta & SM & ss131070541 & 106.48 & $0.110 \pm 0.018$ & $1.33 E-09^{* *}$ & 10 & ss131036304 & ss131562987 \\
\hline 5 & $x$ & $\mathrm{HBF}$ & Meta & SM & ss131070541 & 106.48 & $0.187 \pm 0.021$ & $9.06 \mathrm{E}-19^{* *}$ & 18 & ss131067158 & ss131563051 \\
\hline 5 & $x$ & LBF & Meta & SM & ss131070541 & 106.48 & $0.108 \pm 0.018$ & $1.46 \mathrm{E}-09^{* *}$ & 1 & - & - \\
\hline
\end{tabular}

a We operationally define two loci with the distance between their lead SNPs less than $5 \mathrm{Mb}$ as the same QTL except the loci on chromosome X. All loci close to the recombination cold spot (more than $30 \mathrm{Mb}$, with an extremely low rate of recombination) in the middle of $X$ chromosome was considered the same QTL because of too few SNPs in this region

b Chromosome

c Abbreviations of trait names are in Additional file 1: Table S1

d The position of the unmapped SNP (ss131029816) is deduced by its tightly linked SNP (ss131566312)

e The direction of SNP effect estimated by the meta-GWAS was not shown because the linkage phase may be inconsistent among the four populations, and the SNP effect cannot be estimated by the multi-trait GWAS

$f * * 1 \%$ genome-wide significant; ${ }^{*} 5 \%$ genome-wide significant

$g$ Number of SNPs that surpass the significance level 
in common among the four populations and the four methods.

\section{Results}

\section{Descriptive statistics of the traits}

The descriptive statistics for the nine growth and fatness traits in the $F_{2}$ and Sutai populations were reported in our previous publications [24, 25]. Additional file 1: Table S1 shows the means and standard errors for these nine traits as well as the phenotypic differences between females and males in the Erhualian and Laiwu populations. Growth rates were not significantly different between sexes in the two populations, except that Erhualian males grew faster $(P=0.03)$ than females from 210 to 240 days of age. Backfat thickness at the four localizations was significantly higher $\left(P<10^{-6}\right)$ in males than in females in the Erhualian population, while no significant difference was observed in the Laiwu population. Males deposited more $(P<0.01)$ leaf fat, while females stored more $\left(P<10^{-10}\right)$ fat in the abdomen in both populations. Veil fat was significantly heavier $\left(P=6.77 \times 10^{-6}\right)$ in males than in females in Erhualian pigs, while there was no significant difference in veil fat between sexes in Laiwu pigs.

\section{Phenotypic correlation coefficients between traits}

Raw phenotypic correlation coefficients between the measured traits in the two populations are in Additional file 2: Table S2. All correlation coefficients between the measured traits were significant $(P<0.05)$ in the Erhualian and Laiwu populations, except that between $\mathrm{ADG}_{0-210}$ and $\mathrm{ADG}_{210-240}$ in the Laiwu population. In general, fatness traits were more significantly correlated with each other than growth traits in both populations. $\mathrm{ADG}_{0-210}$ had a moderate positive correlation with the other traits and $\mathrm{ADG}_{210-240}$ showed a weak positive correlation with the other traits.

\section{Qualified SNPs and animals in the GWAS}

All genotyped animals passed quality control with SNP call rates higher than 0.95. A total of 34,636 (4825), 36,341 (4979), 24,602 (3492) and 32,058 (4527) mapped (unmapped) SNPs were qualified for GWAS in the $F_{2}$, Sutai, Erhualian and Laiwu populations, respectively. The $P$ values of the 5\% (suggestive) genome-wide significant threshold were equal to $1.27 \times 10^{-6}\left(2.53 \times 10^{-5}\right)$, $1.21 \times 10^{-6}\left(2.42 \times 10^{-5}\right), 1.78 \times 10^{-6}\left(3.56 \times 10^{-5}\right)$ and $1.37 \times 10^{-6}\left(2.73 \times 10^{-5}\right)$ in these four populations, respectively. Based on the pig genome assembly (Sscrofa10.2, http://www.ensembl.org/Sus_scrofa/Info/ Index), the average physical distances between adjacent SNPs were 74.7, 71.2, 105.1 and $80.7 \mathrm{~kb}$ in these four populations, respectively. A common set of 15,429 qualified SNPs across the four populations was used in the GWAS meta-analysis, with average physical distance between adjacent SNPs of $167.7 \mathrm{~kb}$. The $P$ values of the $5 \%$ genome-wide and suggestive significant thresholds were equal to $3.24 \times 10^{-6}$ and $6.48 \times 10^{-5}$, respectively, in the meta-analysis.

\section{SNPs identified by single-trait GWAS}

Table 1 shows the genome-wide significant regions for the nine fatness and growth traits identified by the four GWAS approaches in this study. The SS-GWAS and SMGWAS identified 15 and 31 chromosomal regions (loci) associated with these nine traits (Table 1; Fig. 1 [see Additional file 3: Table S3, Additional file 4: Figure S1]),

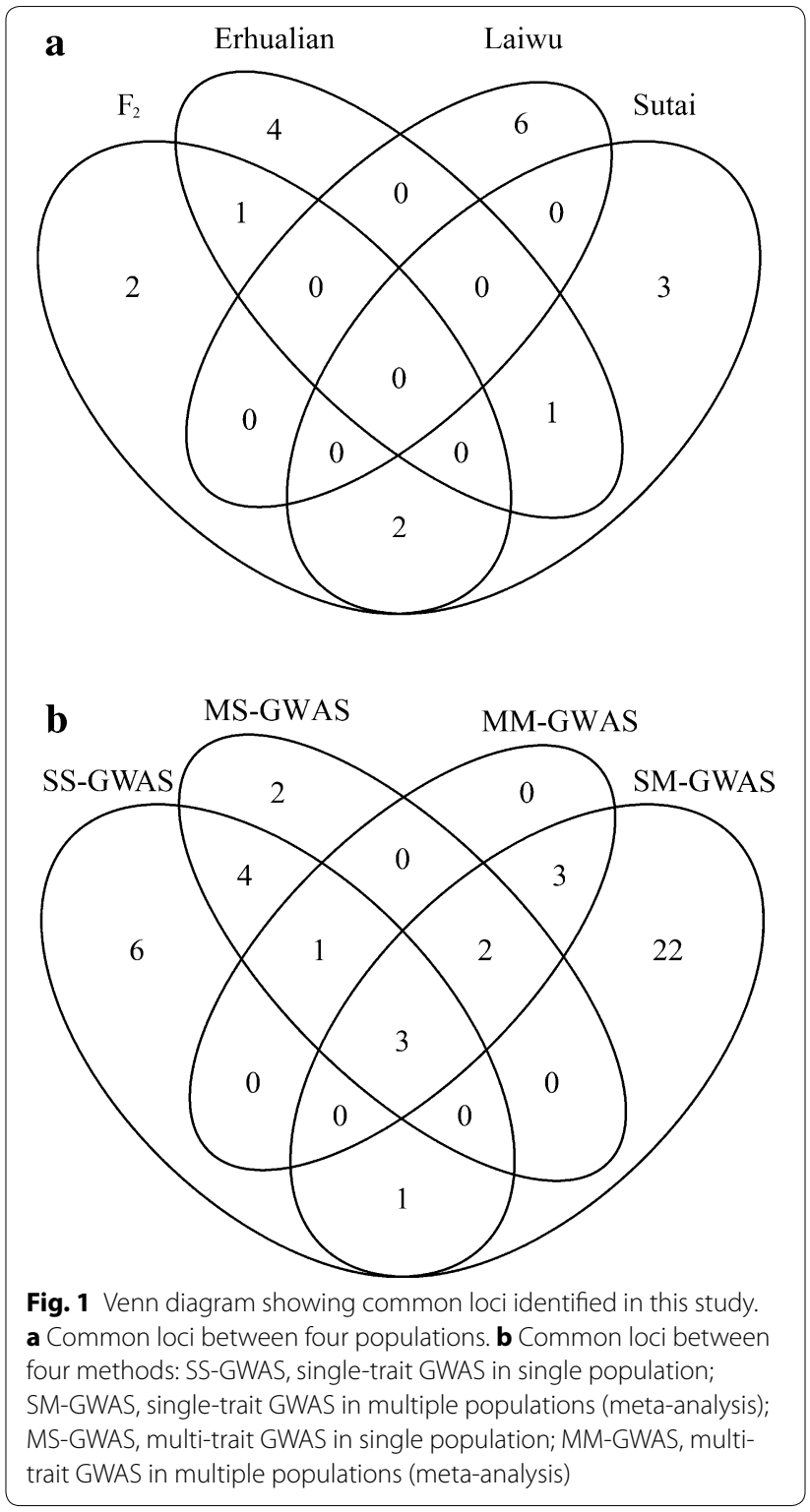


respectively. Four loci were consistently detected by the two methods, one each on SSC1, 2, 7 and X.

\section{SS-GWAS}

In the $F_{2}$ population, the most promising locus was mapped at $34.8 \mathrm{Mb}$ on SSC7 (Table 1). Another genomewide significant locus was identified in the middle of chromosome X. Two suggestive loci were detected, one on SSC1 and one on SSC3 [see Additional file 3: Table S3]. Five and four suggestive loci were identified in the Sutai and Laiwu datasets, respectively. No locus was significant at the genome-wide level in these two datasets. The loci on SSC1, 2 and 8 were detected exclusively for the Sutai population [see Additional file 3: Table S3, Additional file 4: Figure S1]. A genome-wide locus was identified at $34.84 \mathrm{Mb}$ on SSC7 for the Erhualian population. Another three suggestive loci were identified, including two population-specific loci, one each on SSC4 and 6 [see Additional file 3: Table S3, Additional file 4: Figure S1].

\section{SM-GWAS}

The SM-GWAS identified 31 loci that were distributed on all chromosomes except SSC11 and 13, including 22 loci that were not identified in the SS-GWAS and four genome-wide loci, one each on SSC2, 4, 7 and $\mathrm{X}$, that were also identified in the SS-GWAS (Fig. 1; Table 2, and Table S3 [see Additional file 3: Table S3]). These four genome-wide loci were all associated with backfat thickness at the four evaluated sites, and the loci on SSC4 and 7 were also associated with ADG and LFW, respectively.

\section{Loci identified by multi-trait GWAS MS-GWAS}

The MS-GWAS revealed 12 loci associated with both fatness and growth traits, including two loci with genomewide significance (Fig. 2; Table 1; Additional file 3: Table S3). Four loci were identified for the $F_{2}$ population, including two genome-wide significant loci, one at $34.80 \mathrm{Mb}$ on SSC7 and one at $63.65 \mathrm{Mb}$ on chromosome $\mathrm{X}$. For the Sutai population, three loci were identified, including one genome-wide locus at $78.58 \mathrm{Mb}$ on SSCX. For the Laiwu population, only four suggestive loci were identified. Four loci were detected for the Erhualian population, including one genome-wide locus at $34.84 \mathrm{Mb}$ on SSC7 (Fig. 2).

\section{MM-GWAS}

Nine loci were identified in the MM-GWAS, including three genome-wide significant loci on SSC4, 7 and X (Fig. 2). All nine loci were identified by the SM-GWAS, except the suggestive locus at $10.53 \mathrm{Mb}$ on SSC5 (Fig. 1).

\section{Additive effects of top SNPs in the four populations}

The additive effects of top SNPs at genome-wide and suggestive significant levels for the four populations are in Table 2 and see Additional file 5: Table S4, respectively.

Table 2 Genome-wide significant SNPs detected by the meta-analysis of single-trait GWAS common to the four populations, with corresponding additive effects

\begin{tabular}{|c|c|c|c|c|c|c|c|c|}
\hline \multirow[t]{2}{*}{$C h r^{a}$} & \multirow[t]{2}{*}{ Trait $^{\mathbf{b}}$} & \multirow[t]{2}{*}{ Top SNP } & \multirow[t]{2}{*}{ Pos Mb } & \multirow[t]{2}{*}{ Allele } & \multicolumn{4}{|l|}{ Effect $\pm S E^{c}$} \\
\hline & & & & & $F_{2}$ & Sutai & Erhualian & Laiwu \\
\hline 2 & HBF & ss131211507 & 3.61 & A & $0.149 \pm 0.046^{* *}$ & $0.146 \pm 0.039^{* *}$ & $0.051 \pm 0.066$ & $-0.042 \pm 0.121$ \\
\hline 4 & FBF & ss131269439 & 81.71 & A & $-0.148 \pm 0.061^{*}$ & $-0.114 \pm 0.060$ & $-0.234 \pm 0.068^{* *}$ & $-0.198 \pm 0.067^{* *}$ \\
\hline 4 & $\mathrm{HBF}$ & ss131269678 & 82.25 & A & $-0.292 \pm 0.076^{* *}$ & $0.110 \pm 0.046^{*}$ & $0.048 \pm 0.077$ & $-0.256 \pm 0.079^{* *}$ \\
\hline 4 & SBF & ss478935222 & 85.09 & G & $-0.176 \pm 0.063^{* *}$ & $-0.129 \pm 0.050^{*}$ & $0.323 \pm 0.136^{*}$ & $-0.196 \pm 0.087^{*}$ \\
\hline 7 & SBF & ss131343534 & 34.56 & G & $0.420 \pm 0.058^{* *}$ & $0.094 \pm 0.088$ & $-0.083 \pm 0.094$ & $0.020 \pm 0.169$ \\
\hline 7 & FBF & ss131343534 & 34.56 & G & $0.565 \pm 0.062^{* *}$ & $0.097 \pm 0.078$ & $-0.194 \pm 0.088^{*}$ & $-0.082 \pm 0.125$ \\
\hline 7 & $\mathrm{HBF}$ & ss131343534 & 34.56 & G & $0.655 \pm 0.074^{* *}$ & $0.266 \pm 0.085^{* *}$ & $-0.225 \pm 0.089^{*}$ & $0.047 \pm 0.145$ \\
\hline 7 & LFW & ss131344553 & 36.20 & G & $0.325 \pm 0.070^{* *}$ & $0.119 \pm 0.033^{* *}$ & $0.296 \pm 0.092^{* *}$ & $0.146 \pm 0.070^{*}$ \\
\hline 7 & $\mathrm{LBF}$ & ss131347175 & 40.85 & A & $-0.435 \pm 0.062^{* *}$ & $-0.050 \pm 0.033$ & $-0.315 \pm 0.068^{* *}$ & $0.119 \pm 0.054^{*}$ \\
\hline$x$ & SBF & ss131570179 & 46.75 & A & $-0.162 \pm 0.030^{* *}$ & $-0.084 \pm 0.030^{* *}$ & $-0.127 \pm 0.072$ & $-0.137 \pm 0.088$ \\
\hline$x$ & $\mathrm{HBF}$ & ss131070541 & 106.48 & G & $-0.234 \pm 0.026^{* *}$ & $-0.028 \pm 0.054$ & $-0.145 \pm 0.056^{* *}$ & $-0.179 \pm 0.080^{* *}$ \\
\hline$x$ & LBF & ss131070541 & 106.48 & G & $-0.141 \pm 0.023^{* *}$ & $0.029 \pm 0.045$ & $-0.058 \pm 0.048$ & $-0.091 \pm 0.064$ \\
\hline$x$ & FBF & ss131070541 & 106.48 & G & $-0.131 \pm 0.022^{* *}$ & $-0.051 \pm 0.052$ & $-0.057 \pm 0.055$ & $-0.079 \pm 0.070$ \\
\hline
\end{tabular}

a Chromosome

b Abbreviations of trait names are in Additional file 1:Table S1

c The significant level is a single point without Bonferroni correction: ** $P \leq 0.01$; $P \leq 0.05$ 

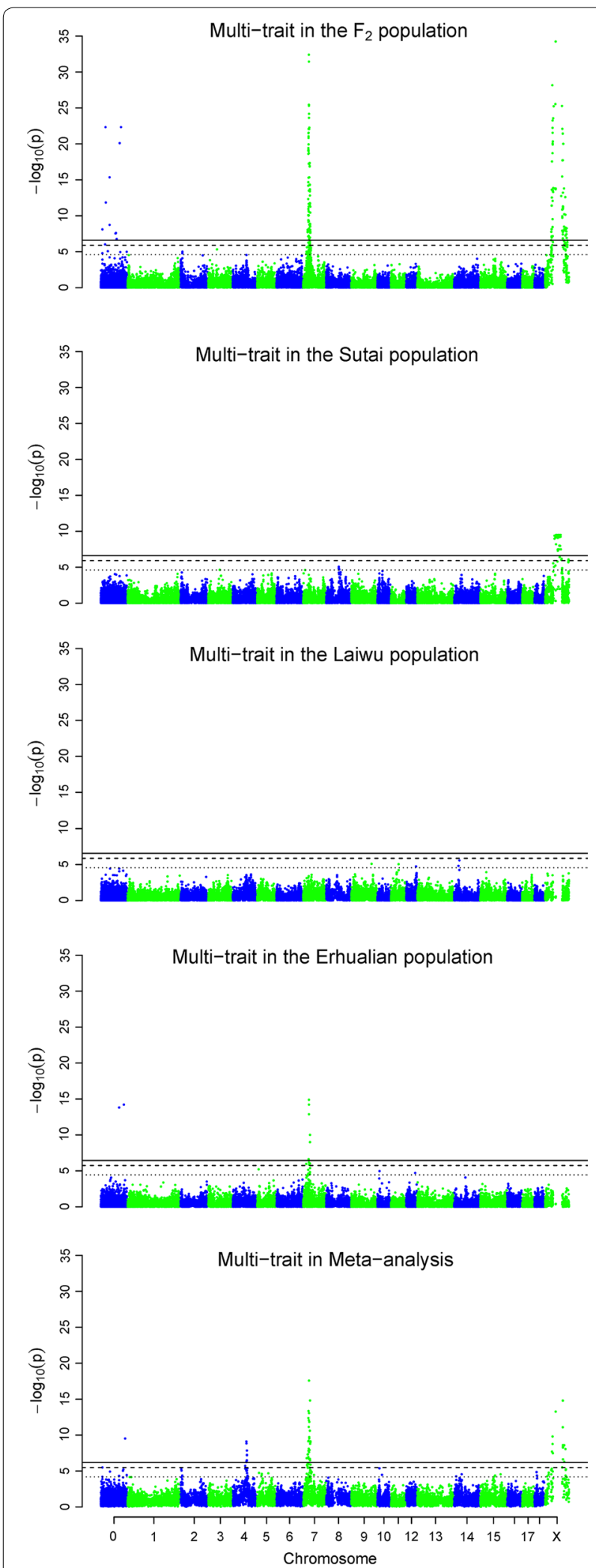

Fig. 2 Manhattan plots of the multi-trait GWAS and meta-analysis for fatness and growth traits in $\mathrm{F}_{2}$, Sutai, Laiwu and Erhualian populations. The solid, dashed and dotted horizontal lines indicate the 1 and 5\% genome-wide and suggestive significant threshold values, respectively. Unmapped SNPs are assigned on chromosome 0 and arbitrary ordered by their names
Thirteen SNPs were associated with fatness traits at the genome-wide significant level in the SM-GWAS. Among these 13 SNPs, five had a consistent direction of additive effects across the four populations, while five showed inconsistent effect directions. The other three SNPs just achieved the significance level for one population. We observed a similar pattern for suggestive SNPs: 12 consistent SNPs and nine inconsistent SNPs. This indicates that the linkage phases between these SNPs and the causative mutations that underlie the detected QTL are not always identical across populations.

\section{Results of the conditional GWAS}

We identified two loci associated with multiple traits at the genome-wide significant level by the MS-GWAS. One region was located on SSC7 (between 34.80 and $34.84 \mathrm{Mb}$ ) for the $\mathrm{F}_{2}$ and Erhualian populations, and the other mapped to SSCX (between 63.65 and $66.25 \mathrm{Mb}$ ) for the $F_{2}$ and Sutai populations. After fixing the effects of the top SNPs (detected by the MS-GWAS) in the SSGWAS model, we found that no SNP on SSC7 showed any association signal with multiple traits for both the $\mathrm{F}_{2}$ and Erhualian populations (Fig. 3a, b). No SNP was significantly associated with the nine traits in the region between 60 and $90 \mathrm{Mb}$ on SSCX for the $\mathrm{F}_{2}$ and Sutai populations, respectively (Fig. 3c, d).

\section{Discussion}

Comparison of our results with previously reported loci Previously, we had identified 15 significant loci on 11 chromosomes for the traits measured in the $F_{2}$ and Sutai populations that were also analysed here [25]. In the current study, we identified nine loci on six chromosomes for the same traits by single-trait and multi-trait GWAS in these two populations. Only four loci (one each on SSC2, 4, 7 and X, see Tables 1, 2) were common between both studies and have also been consistently reported in previous studies [2-4, 6, 24, 25]. The lower power in the current study can be attributed to the fact that residual inflation was not corrected in our previous study, but was corrected by genomic control here. To date, 1880 QTL for growth and 1070 for fatness traits have been reported in the pig QTL database [26]. Although we conducted GWAS on four divergent populations using four approaches, no novel loci were identified in the current study. The four GWAS methods yielded different results with few common results, which is likely due to the fact that each method has its own advantage to identify distinct loci. For example, the multi-trait analysis is suitable for detecting pleiotropic loci, the meta-analysis is sufficiently powerful to identify common loci across populations, and the single-population GWAS is an effective method to detect population-specific loci. 

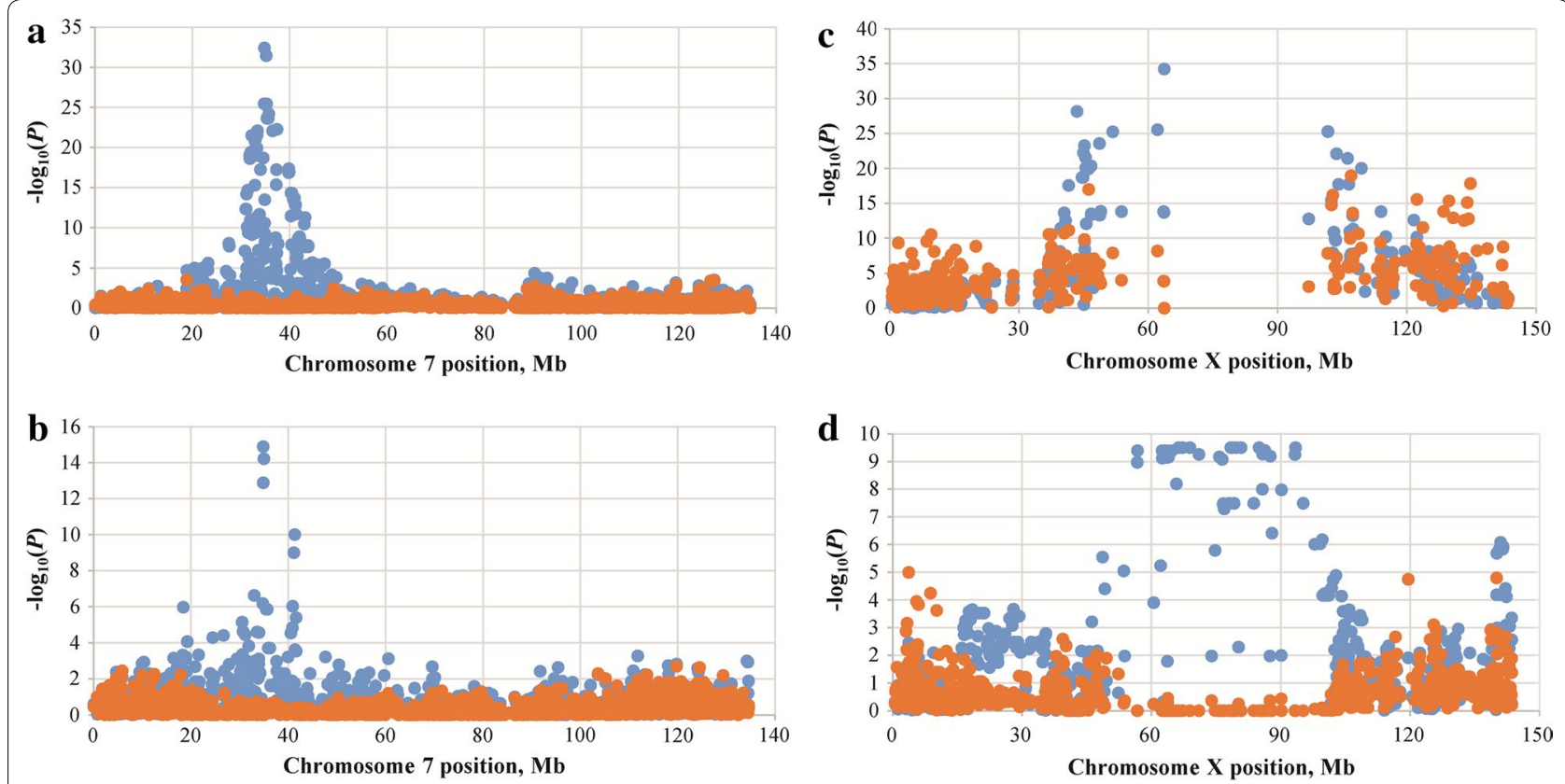

Fig. $3 \log _{10}(P)$ of the multi-trait test before (blue) and after (orange) fitting the top SNPs at the loci with genome-wide significance. a Chromosome 7 in the $F_{2}$ population. b Chromosome 7 in the Erhualian population. c Chromosome $X$ in the $F_{2}$ population. d Chromosome $X$ in the Sutai population

\section{Unmapped SNPs improve the power of association analyses}

As shown in our previous studies [13, 27, 28], retaining the unmapped SNPs can improve the power of GWAS. In this study, we identified 18 unmapped SNPs that surpassed the suggestive or genome-wide significant level [see Additional file 4: Figure S1]. By applying linkage analysis in the $F_{2}$ population, we deduced the approximate genomic locations of 15 unmapped SNPs [see Additional file 6: Table S5], and the use of these SNPs improved the power of the current GWAS. For example, we did not identify any mapped SNPs associated with $\mathrm{ADG}_{210-240}$ in the Sutai dataset. However, a SNP (ss131031851) that had two locations (65.29 and $65.38 \mathrm{Mb})$ on SSC10 displayed an association signal with $\mathrm{ADG}_{210-240}$ [see Additional file 4: Figure S1, Additional file 6: Table S5]. The unmapped SNP ss131029816 is another example. We detected only one mapped SNP on SSC6 that was associated with $\mathrm{ADG}_{210-240}$ at a suggestive significant level in the SM-GWAS, while the unmapped SNP ss131029816 showed a genome-wide significant association with this trait [see Additional file 4: Figure S1, Additional file 6: Table S5]. In a two-point linkage analysis on the $\mathrm{F}_{2}$ population, ss131029816 was tightly linked $(\theta=0.0022)$ to ss131566312 at 71.6 Mb on SSC6. Therefore, a genomewide significant locus for $\mathrm{ADG}_{210-240}$ was identified at around 71.6 Mb on SSC6.

\section{Single-population GWAS versus meta-analysis}

The SS-GWAS showed that four loci were common to two populations (Fig. 1a). Two loci, one at the proximal end of SSC2 and one in the middle of SSCX, were shared between the $\mathrm{F}_{2}$ and Sutai populations (Table 1 and Table S3 [see Additional file 3: Table S3]). The locus around $34.80 \mathrm{Mb}$ on SSC7 was associated with fatness traits in both the $\mathrm{F}_{2}$ and Erhualian datasets (Table 1). This locus may be segregating in the population of Laiwu pigs since we observed a weak signal around it (Fig. 1; see Additional file 4: Figure S1). Another locus in the middle of SSC12 was found to be shared between the Erhualian and Laiwu populations. The segregation of these common loci in multiple populations should allow us to efficiently fine map these loci by using higher-density chips, such as the $600 \mathrm{~K}$ SNP chip, since inter-population linkage disequilibrium usually extends over short distances $\left(r_{0.3}^{2}=10.5 \mathrm{~kb}\right)$ in Chinese pigs [29].

We noted that there were no common loci across three or four populations in the SS-GWAS. However, the SMGWAS identified 31 significant loci that were putative common loci across these four populations (Fig. 1b). One explanation for the discrepancy between the two GWAS methods is that the detection power of SS-GWAS is lower than that of SM-GWAS because of the small sizes of individual populations, which prevents the detection of these putative common loci. Another possibility is that some informative SNPs in the SS-GWAS were deleted during the filtering process in the SM-GWAS. Of the 31 

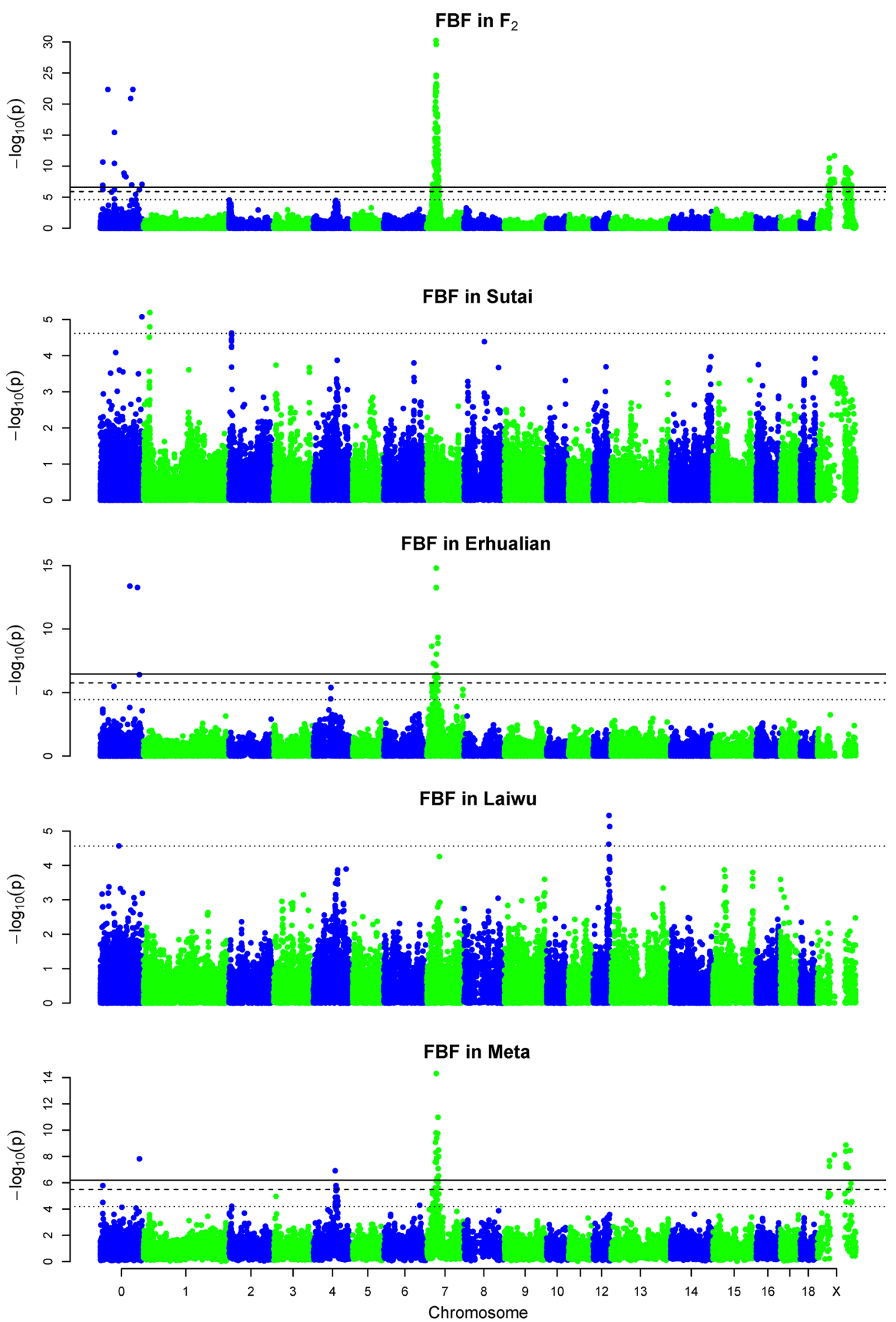

Fig. 4 Manhattan plots of the single-trait GWAS and meta-analysis for backfat thickness at first rib (FBF) in $F_{2}$, Sutai, Laiwu and Erhualian populations. The solid, dashed and dotted horizontal lines indicate the 1 and 5\% genome-wide and suggestive significant threshold values, respectively. Unmapped SNPs are assigned on chromosome 0 and arbitrary ordered by their names 
loci, 13 surpassed the genome-wide significant level. It is interesting that all 13 SNPs had 'single point significant' effects in the $F_{2}$ population, while only some of these SNPs showed such effects in the other three populations (Table 2). This is most likely due to the fact that the $F_{2}$ population has the largest population size and thus had greater impact on the results of the meta-analysis.

It is known that meta-analysis of GWAS can improve the detection power when two or more populations show consistent association signals with target traits. For example, we detected a suggestive locus for FBF at 65.72 Mb on SSC4 in the Erhualian population [see Additional file 3: Table S3], while this region had only a weak (non significant) association signal with FBF in the other three populations (Fig. 4). By performing a meta-analysis across the four populations, we observed an association signal at the $1 \%$ genome-wide significant level in this region (Fig. 4; Table 1). However, population-specific loci could not be detected by the meta-analysis. For instance, we identified an $F_{2}$-specific locus for $A_{D G} G_{210-240}$ at $53.06 \mathrm{Mb}$ on SSC3. This locus was not detected by the meta-analysis.

\section{Single-trait GWAS versus multi-trait GWAS}

If a locus has pleiotropic effects on multiple traits, the multi-trait method can be used to enhance the power of GWAS. For example, the SS-GWAS identified 15 loci in this study, more than half of which were also identified by the MS-GWAS (Fig. 1b). In the SS-GWAS, the minimum $P$ values at the locus on SSC7 for the $\mathrm{F}_{2}$ and Erhualian populations were $1.75 \mathrm{E}-18$ and $6.96 \mathrm{E}-14$, respectively; whereas these two values decreased to $3.97 \mathrm{E}-33$ and $1.29 \mathrm{E}-15$ in the MS-GWAS, respectively. In contrast, if a locus has no pleiotropic effects, the MSGWAS can decrease the signal. For instance, the suggestive locus at $303.92 \mathrm{Mb}$ on SSC1 for $\mathrm{ADG}_{0-210}$ in the $\mathrm{F}_{2}$ population was identified by the SS-GWAS but not by the MS-GWAS. Furthermore, it has been reported that multi-trait GWAS can map the locus more accurately than the single-trait method when the marker density is high [11]. It should be noted that the extent of linkage disequilibrium is usually large in $F_{2}$ populations, for which a multi-trait GWAS based on medium-density SNP chips, such as $60 \mathrm{~K}$ SNPs, is expected to have high detection power.

\section{Linkage versus pleiotropy}

By applying multi-trait GWAS, we detected three prominent loci on SSC4, 7 and X (Fig. 2), respectively. The detection of these loci could be caused by pleiotropy or closely-linked causal QTL. The SSC7 locus detected for the $F_{2}$ and Erhualian populations, as well as the SSCX locus for the Sutai pigs (Fig. 2), are most likely loci with pleiotropic effects, since we found no SNP that showed an association signal with the nine traits when the top SNPs were fixed in the MS-GWAS model (Fig. 3a, b, d). After correction for the effect of the top SNP on SSCX, several SNPs were still associated with the traits tested in $\mathrm{F}_{2}$ pigs (Fig. 3c). This implies that the locus on SSCX could be caused by closely-linked causal variants in $\mathrm{F}_{2}$ pigs.

\section{Conclusions}

In this study, we explored four GWAS approaches to identify genomic loci for nine growth and fatness traits in four pig populations. Compared to the single-trait analysis, the meta-analysis had less power to identify population-specific loci but more power to detect population-shared loci. Compared to the single-trait analysis, the multiple-trait analysis reduced the power to detect trait-specific loci but enhanced the power to identify the common loci across traits. Our findings demonstrate that the meta-analysis and the multi-trait method can be used to increase the power of GWAS.

\section{Additional files}

Additional file 1: Table S1. Descriptive statistics and differences between females and males for nine growth and fatness traits in the Erhualian and Laiwu pigs.

Additional file 2: Table S2. Phenotypic correlation coefficients between nine growth and fatness traits in the Laiwu and Erhualian pigs.

Additional file 3: Table S3. The suggestive significant loci for nine fatness and growth traits identified in this study.

Additional file 4: Fig. S1. Manhattan plots of the single-trait GWAS and meta-analysis for nine fatness and growth traits in the $F_{2}$, Sutai, Laiwu and Erhualian populations. The solid, dashed and dotted horizontal lines indicate the $1 \%$ and $5 \%$ genome-wide and suggestive significant threshold values, respectively. Unmapped SNPs are assigned on chromosome 0 and arbitrary ordered by their names.

Additional file 5: Table S4. The additive effects of suggestive significant SNPs detected by the meta-analysis in the four populations.

Additional file 6: Table S5. Tight linkage of unassigned significant SNPS with the mapped SNPS. This table shows approximate genomic positions of the unassigned significant SNPs by a two-point linkage analysis in the $\mathrm{F}_{2}$ population.

\section{Authors' contributions}

LSH conceived and designed the experiment. YG and JR analyzed the data. YG, $\mathrm{YH}$ and $\mathrm{LJH}$ performed the experiment. JM, CC and HA contributed materials. JR and YG wrote the manuscript. All authors read and approved the final manuscript.

\section{Acknowledgements}

This research was supported by the National Natural Science Foundation of China $(31525023,31460590,31660303)$ and Jiangxi province (20142BAB204017) to JR and YG.

\section{Competing interests}

The authors declare that they have no competing interests.

Received: 31 December 2015 Accepted: 6 February 2017

Published online: 14 February 2017 


\section{References}

1. Miller ER, Ullrey DE. The pig as a model for human nutrition. Annu Rev Nutr. 1987;7:361-82.

2. Bidanel JP, Milan D, lannuccelli N, Amigues Y, Boscher MY, Bourgeois F, et al. Detection of quantitative trait loci for growth and fatness in pigs. Genet Sel Evol. 2001;33:289-309.

3. Walling GA, Visscher PM, Andersson L, Rothschild MF, Wang L, Moser G, et al. Combined analyses of data from quantitative trait loci mapping studies. Chromosome 4 effects on porcine growth and fatness. Genetics. 2000;155:1369-78.

4. Rohrer GA. Identification of quantitative trait loci affecting birth characters and accumulation of backfat and weight in a Meishan-White Composite resource population. J Anim Sci. 2000;78:2547-53.

5. Sato S, Oyamada Y, Atsuji K, Nade T, Kobayashi E, Mitsuhashi T, et al. Quantitative trait loci analysis for growth and carcass traits in a Meishan × Duroc F2 resource population. J Anim Sci. 2003;81:2938-49.

6. Andersson L, Haley CS, Ellegren H, Knott SA, Johansson M, Andersson K, et al. Genetic mapping of quantitative trait loci for growth and fatness in pigs. Science. 1994;263:1771-4.

7. Ramos AM, Crooijmans RP, Affara NA, Amaral AJ, Archibald AL, Beever JE, et al. Design of a high density SNP genotyping assay in the pig using SNPs identified and characterized by next generation sequencing technology. PLoS One. 2009;4:e6524.

8. Evangelou E, loannidis JP. Meta-analysis methods for genome-wide association studies and beyond. Nat Rev Genet. 2013;14:379-89.

9. Korol AB, Ronin YI, Itskovich AM, Peng J, Nevo E. Enhanced efficiency of quantitative trait loci mapping analysis based on multivariate complexes of quantitative traits. Genetics. 2001;157:1789-803.

10. Knott SA, Haley CS. Multitrait least squares for quantitative trait loci detection. Genetics. 2000;156:899-911.

11. Bolormaa S, Pryce JE, Reverter A, Zhang Y, Barendse W, Kemper K, et al. A multi-trait, meta-analysis for detecting pleiotropic polymorphisms for stature, fatness and reproduction in beef cattle. PLoS Genet. 2014;10:e1004198.

12. Guo Y, Mao H, Ren J, Yan X, Duan Y, Yang G, et al. A linkage map of the porcine genome from a large-scale White Duroc $\times$ Erhualian resource population and evaluation of factors affecting recombination rates. Anim Genet. 2009;40:47-52

13. Guo YM, Zhang XF, Ren J, Ai HS, Ma JW, Huang LS. A joint genome-wide association analysis of pig leg weakness and its related traits in an F2 population and a Sutai population. J Anim Sci. 2013;91:4060-8.

14. Wang L, Wang A, Wang L, Li K, Yang G, He R, et al. Animal genetic resources in China: pigs. Beijing: China Agricultural Press; 2011.
15. Astle W, Balding DJ. Population structure and cryptic relatedness in genetic association studies. Stat Sci. 2009;24:451-71.

16. Amin N, van Duijn CM, Aulchenko YS. A genomic background based method for association analysis in related individuals. PLoS One. 2007;2:e1274.

17. Lander E, Kruglyak L. Genetic dissection of complex traits: guidelines for interpreting and reporting linkage results. Nat Genet. 1995;11:241-7.

18. Chen WM, Abecasis GR. Family-based association tests for genomewide association scans. Am J Hum Genet. 2007:81:913-26.

19. Devlin B, Roeder K. Genomic control for association studies. Biometrics. 1999:55:997-1004.

20. Zheng G, Freidlin B, Li Z, Gastwirth JL. Genomic control for association studies under various genetic models. Biometrics. 2005;61:186-92.

21. Green P, Falls K, Crooks S. Cri-map Version 2.4. St. Louis: Washington University School of Medicine; 1994.

22. Sobel E, Lange K. Descent graphs in pedigree analysis: applications to haplotyping, location scores, and marker-sharing statistics. Am J Hum Genet. 1996:58:1323-37.

23. Barrett JC, Fry B, Maller J, Daly MJ. Haploview: analysis and visualization of LD and haplotype maps. Bioinformatics. 2005;21:263-5.

24. Ai H, Ren J, Zhang Z, Ma J, Guo Y, Yang B, et al. Detection of quantitative trait loci for growth- and fatness-related traits in a large-scale White Duroc $\times$ Erhualian intercross pig population. Anim Genet. 2012;43:383-91.

25. Qiao RM, Gao J, Zhang ZY, Lin L, Xie XH, Fan Y, et al. Genome-wide association analyses reveal significant loci and strong candidate genes for growth and fatness traits in two pig populations. Genet Sel Evol. 2015;47:17.

26. Hu ZL, Park CA, Wu XL, Reecy JM. Animal QTLdb: an improved database tool for livestock animal QTL/association data dissemination in the postgenome era. Nucleic Acids Res. 2013:41:D871-9.

27. Guo Y, Hou L, Zhang X, Huang M, Mao H, Chen H, et al. A meta analysis of genome-wide association studies for limb bone lengths in four pig populations. BMC Genet. 2015;16:95.

28. Guo YM, Zhang ZY, Ma JW, Ai HS, Ren J, Huang LS. A genomewide association study of feed efficiency and feeding behaviors at two fattening stages in a White Duroc $\times$ Erhualian F population. J Anim Sci. 2015;93:1481-9.

29. Ai H, Huang L, Ren J. Genetic diversity, linkage disequilibrium and selection signatures in chinese and Western pigs revealed by genome-wide SNP markers. PLoS One. 2013;8:e56001.

\section{Submit your next manuscript to BioMed Central and we will help you at every step:}

- We accept pre-submission inquiries

- Our selector tool helps you to find the most relevant journal

- We provide round the clock customer support

- Convenient online submission

- Thorough peer review

- Inclusion in PubMed and all major indexing services

- Maximum visibility for your research

Submit your manuscript at www.biomedcentral.com/submit
BioMed Central 